Brief Communication

\title{
Linking membrane physical properties and low temperature tolerance in arthropods ${ }^{\text {is }}$
}

\author{
Dorthe Waagner $^{\mathrm{a}, *}$, Hélène Bouvrais ${ }^{\mathrm{b}}$, John H. Ipsen ${ }^{\mathrm{b}}$, Martin Holmstrup ${ }^{\mathrm{a}}$ \\ a Aarhus University, Department of Bioscience, Vejlsøvej 25, DK-8600 Silkeborg, Denmark \\ ${ }^{\mathrm{b}}$ MEMPHYS - Center for Biomembrane Physics, Department of Physics, Chemistry and Pharmacy, University of Southern Denmark, Campusvej 55, 5230 Odense M, Denmark
}

\section{A R T I C L E I N F O}

\section{Article history:}

Received 2 July 2013

Accepted 18 September 2013

Available online 27 September 2013

\section{Keywords:}

Arthropod

Bending rigidity

Collembola

Cold tolerance

Ectotherm

GUV

Membrane fluidity

\begin{abstract}
A B S T R A C T
Maintenance of membrane fluidity is of crucial importance in ectotherms experiencing thermal changes. This maintenance has in ectotherms most often been indicated using indirect measures of biochemical changes of phospholipid membranes, which is then assumed to modulate the physico-chemical properties of the membrane. Here, we measure bending rigidity characterizing the membrane flexibility of reconstituted membrane vesicles to provide a more direct link between membrane physical characteristics and low temperature tolerance. Bending rigidity of lipid bilayers was measured in vitro using Giant Unilamellar Vesicles formed from phospholipid extracts of the springtail, Folsomia candida. The bending rigidity of these membranes decreased when exposed to $0.4 \mathrm{vol} \%$ ethanol $(0.23 \mathrm{mM} / \mathrm{L})$. Springtails exposed to ethanol for $24 \mathrm{~h}$ significantly increased their cold shock tolerance. Thus, by chemically inducing decreased membrane rigidity, we have shown a direct link between the physico-chemical properties of the membranes and the capacity to tolerate low temperature in a chill-susceptible arthropod.

(c) 2013 Elsevier Inc. All rights reserved.
\end{abstract}

Biological membranes accommodate multiple functions such as barrier between compartments and as particular solvent for membrane components. In fully functional cells, membranes are in a fluid, liquid-crystalline phase, where the phospholipids display high degree of acyl-chain conformational and lateral disorder to maintain their biological function. But when biological membranes are cooled, their functions may be impaired through ordering processes where lateral mobility of membrane components is diminished [10]. Furthermore, cooling induces (i) increased hydrophobic thickness of the lipid membrane which strongly perturbs the activity of integral membrane enzymes such as the ion transporters $\mathrm{Na}^{+}, \mathrm{K}^{+}$-ATPase and $\mathrm{Ca}^{2+}$-ATPase $[9,11]$ and (ii) increased occurrence of gel-phase domains leading to damaging loss of the barrier function [4]. These perturbations can be avoided by adaptive changes in the composition of membrane phospholipids helping to preserve appropriate physico-chemical properties of the membrane under changing temperatures. This is a common phenomenon in ectothermic animals, known as "homeoviscous adaptation" or "homeophasic adaptation" [6]. Homeoviscous adaptation relies mainly on biochemical modifications such as changes in: (i) composition of phospholipid head groups, (ii) position of phospholipid fatty acids (PLFAs) on the phospholipid head groups,

\footnotetext{
Statement of funding: This study was supported by The Danish Council for Independent Research, contract no. 10-084579.

* Corresponding author.

E-mail addresses: dorthe.waagner@gmail.com, dowa@dmu.dk (D. Waagner).
}

and (iii) composition of PLFAs, for instance increased degree of unsaturation, which may all contribute to increased cold tolerance [6]. However, changed membrane phospholipid compositions are non-direct, indicative measures of changed membrane properties. Direct measurements can be conducted by techniques based on molecular probes sensing a local environment of the membrane (e.g. electron paramagnetic resonance techniques, fluorescence polarization measurements and deuterium NMR). But since the biological membrane is a very complex many-component system with various mesoscopic environments, e.g. lateral domains and annular lipids [9], such experimental procedures are questionable for characterizing the overall state of the membrane. To overcome this problem, we have recently developed a measure for such characterization based on the mechanical stiffness of the membrane. We showed that it is possible to form stable bilayer vesicles from the phospholipid extract of invertebrates and measure the macroscopic membrane mechanical modulus, the bending rigidity of these vesicles [1]. This measure of the physico-chemical properties of fluid membranes thus reflects bulk characteristics even for a very complex membrane. The bending rigidity expresses the elastic flexibility of the fluid membrane and is thus of key importance in a multitude of cellular processes like membrane adhesion and shaping of cell organelles. Furthermore, bending rigidity has proven to be very sensitive to the exposure of membrane perturbants [1].

It is well-established from simple lipid bilayer studies that chemical compounds interacting with the membrane can have major effects on membrane physico-chemical properties. For example, 
the large group of compounds with anaesthetic action modifies the membranes by adsorption to or partitioning into the phospholipid bilayer resulting in changes of the lipid packing, lipid chain order profile and gel to fluid transition temperature [2]. Molecular dynamic modeling of the effect of ethanol supports previous findings of increased ion permeability, reduced lipid chain order parameter and bilayer instability induced by ethanol [12]. Thus, it may be hypothesized that the disordering effect of ethanol on the membrane can counteract some of the low-temperature induced ordering processes and thus increase the organism cold tolerance without changing the membrane phospholipid composition. This hypothesis was tested using the chill-susceptible springtail, Folsomia candida, as model organism.

$F$. candida used in this study were from a laboratory culture kept at $20^{\circ} \mathrm{C}\left( \pm 1^{\circ} \mathrm{C}\right)$ and $12: 12 \mathrm{~h}$ light cycle on Petri dishes with water saturated Paris of plaster mixed with charcoal. The springtails were fed dried baker's yeast and were $6-8$ weeks old with a fresh weight in the range 150-200 $\mu \mathrm{g}$ when used for experiments.

The effect of sublethal ethanol exposure on cold tolerance in $F$. candida was assessed as survival after a short-term exposure to cold shock. Ethanol uptake was achieved by placing collembolans on the surface of an ethanol/demineralized water solution in air tight containers $\left(4 \mathrm{~cm}\right.$ high and $7 \mathrm{~cm}$ in diameter) at $20^{\circ} \mathrm{C}\left( \pm 1^{\circ} \mathrm{C}\right)$ for $24 \mathrm{~h}$. Ethanol concentrations of 0 (water only; control), 0.1, $0.2,0.3,0.4$ and $0.5 \mathrm{vol} \%$ were used. Live springtails were replicate-wise transferred from ethanol solution to $2 \mathrm{~mL}$ centrifuge tubes. Duration of transfer was less than a minute per replicate, and 5 replicates were used for each treatment. Cold shock $\left(-5.5^{\circ} \mathrm{C}\right)$ for $2 \mathrm{~h}$ was applied in a temperature controlled bath with a precision of $\pm 0.05^{\circ} \mathrm{C}$ (Lauda Eco RE 1050, VWR Bie and Berntsen A/S, Herlev, Denmark). After cold treatment, springtails were allowed to recover on moistened plaster of Paris-charcoal Petri dishes at $20^{\circ} \mathrm{C}\left( \pm 1^{\circ} \mathrm{C}\right)$ for $24 \mathrm{~h}$ before survival was assessed as ability to walk in a coordinated manner.

Giant Unilamellar Vesicles (GUVs) were produced using wholebody phospholipid extracts of $F$. candida. Extraction procedures, membrane phospholipid fatty acid composition of $F$. candida and details of GUV formation are described in detail by Bouvrais et al. [1]. Briefly, phospholipids were extracted from macerated specimens using a modified Bligh-Dyer single-phase lipid extraction. Following phase separation, the chloroform layer was transferred to a test tube and evaporated to dryness under $\mathrm{N}_{2}$. Phospholipids were then isolated from the crude lipid extract by solid-phase extraction (100 mg silicic acid; Isolute, Mid Glamorgan, UK). Polar lipids (mainly membrane phospholipids) were eluted with $1.5 \mathrm{ml}$ methanol and collected in a test tube. About $1 \mathrm{mg}$ dry mass phospholipids were obtained from $100 \mathrm{mg}$ dry mass animal tissue. Since cholesterol was removed from the lipid fraction during the extraction procedure, the missing quantity was introduced as a given volume of cholesterol solution in chloroform (Avanti lipids) to get cholesterol molar ratio of $9.0 \%$.

GUVs were prepared using an electroformation method, where small unilamellar vesicles (SUVs) composed from the springtail phospholipid extracts were deposited on electrodes which after water evaporation were immersed in water previously introduced in a glass cell. An electric field was applied to the electrodes inducing formation of vesicles with a diameter between 10 and $50 \mu \mathrm{m}$ (GUVs) after a few hours.

Bending rigidity of GUVs with pure water or ethanol was measured from observation of the shape deformation and analysis of the thermal fluctuations. Measurements were made for two treatments; i.e. GUVs observed in pure water or a $0.4 \%(\mathrm{v} / \mathrm{v})$ ethanol solution mimicking one of the treatments that springtails were exposed to. We tested the effect of only one ethanol concentration ( $0.4 \mathrm{vol} \%$ ) on bending rigidity since the workload associated with this is substantial. The final bending rigidity values were based on 8 measurements on GUVs in pure water and 11 measurements on GUVs in ethanol solution. ANOVA was used to test the effect of ethanol on GUV bending rigidity. Contingency tables and chisquare analysis were used to test for effect of ethanol on survival rates of $F$. candida. Statistical analyses were conducted using SAS ${ }^{\circledR}$ Enterprise Guide, 4.3 (SAS Institute, Cary, USA) using a significance level at $5 \%$.

We observed that ethanol solution significantly reduced GUV bending rigidity compared to pure water (One-way ANOVA, $\left.F_{(1,18)}=11.26, p=0.004\right)$. For comparison, it should be noted that small variations in the length and composition of the acyl chains result in changes of the bending rigidity that are of a few $k_{B} T$, bending rigidity being equal to $32.2 k_{B} T$ and $37.1 k_{B} T$ for dimyristoyl phosphatidylcholine (DMPC; $14 \mathrm{C}$ atoms in the acyl chains) and dipalmitoyl phosphatidyl choline (DPPC; $16 \mathrm{C}$ atoms in the acyl chains), respectively [5]. Thus, the change in bending rigidity from 20.5 (sem: \pm 0.19 ) to 19.2 (sem: \pm 0.30$) k_{B} T$ caused by $0.4 \mathrm{vol} \%$ ethanol is in the same range as would typically ensue after compositional changes of the membrane phospholipids during cold acclimation of arthropods in the laboratory or during winter acclimatization under field conditions. In the present study, it was assumed that concentrations of ethanol in F. candida body fluids during the $24 \mathrm{~h}$ exposure would reach equilibrium concentrations similar to the external medium since ethanol can rapidly pass across the skin and has an octanol-water partition coefficient $\left(K_{o w}\right)$ close to unity [8]. Thus, a bending rigidity reduction similar to that observed in the GUVs reconstituted from phospholipid extracts of $F$. candida was expected in whole-organisms exposed to ethanol.

Ethanol had significant effect on cold shock tolerance in $F$. candida $\left(\chi^{2}=29.8 ; p<0.0001\right.$; d.f. $=5$; Fig. 1$)$. Intermediate ethanol concentrations increased cold shock survival from $45 \%$ in controls to $65 \%$ at $0.3-0.4 \mathrm{vol} \%$. This implies that the chemically induced decrease of membrane bending rigidity is indeed important for tolerance of low temperature extremes in our model organism. Thus the present report provides indications of a close link between membrane physical properties and thermal biology in arthropods. We used ethanol as an extrinsic agent softening the "average" membrane of the intact organism without changing the composition of membrane phospholipids. This effect translated into an improvement of cold shock tolerance at intermediate ethanol concentrations (0.3-0.4 vol\%) as predicted.

In whole organisms, cold tolerance does not depend on membrane physics alone. Protective responses such as activation of heat

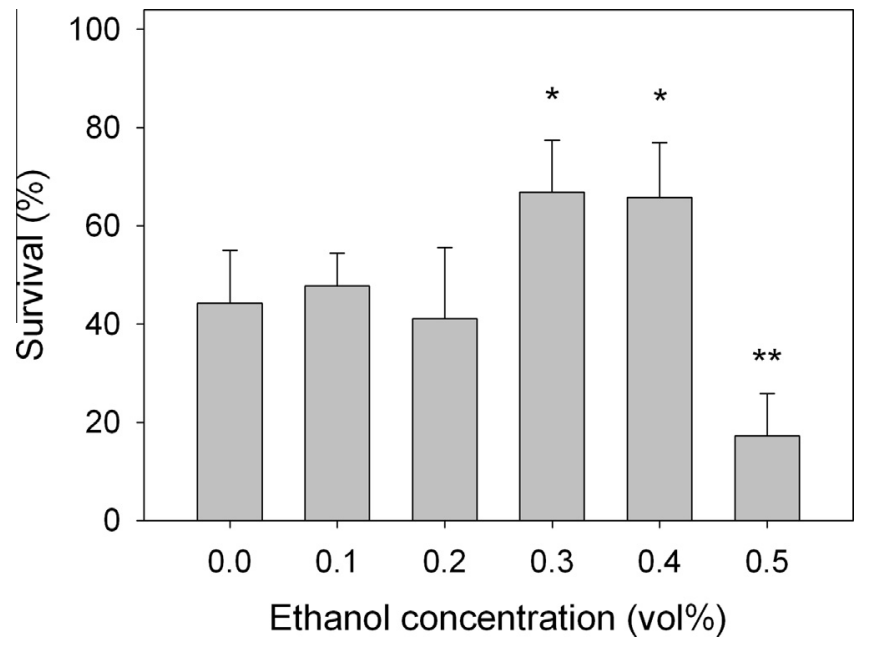

Fig. 1. Effects of ethanol exposure $\left(24 \mathrm{~h}\right.$ at $\left.20^{\circ} \mathrm{C}\right)$ on cold shock $\left(2 \mathrm{~h}\right.$ at $-5.5^{\circ} \mathrm{C}$ ) survival of Folsomia candida. Means \pm s.e.m. $(N=5)$ are shown. Asterisks indicate significant effect of treatment $\left({ }^{*} p \leqslant 0.05 ;{ }^{* *} p \leqslant 0.01\right)$. 
shock proteins (HSPs) are also important in cold tolerance of various organisms [3]. Since increased activation of HSPs can be induced by ethanol [7], the positive effect of the ethanol-induced membrane softening described here may not be the only explanation to increased cold tolerance in F. candida. Activation of Hsp10, Hsp23, Hsp40 and Hsc70 seems to induce increased cold tolerance in $F$. candida [13], but further studies are needed to elucidate the correlation of membrane softening and HSP responses in collembolan cold tolerance.

Ethanol does also have negative impact on survival and cold tolerance of $F$. candida. In concentrations higher than $0.5 \mathrm{vol} \%$, ethanol caused lethality in pilot studies. Thus, the observed negative effect of $0.5 \mathrm{vol} \%$ ethanol on cold tolerance probably represents a situation where the negative effects of ethanol overshadows the beneficial effects for cold tolerance. It may be that the increased ethanol concentration perturbs the suggested HSP response in $F$. candida like in the honeybee, Apis mellifera. There, ethanol at concentrations $\leqslant 5 \%$ induces HSP70. However, when the ethanol concentration in the honeybees increases above $5 \%$ the protective response ceases and along with that the honeybee social behavior is impaired [7]. In the present study, other negative effects of increased ethanol concentration could be decreased ordering of PLFA chains and profound perturbation of protein structures [2,12]. The latter possibly due to changed lateral pressure profile exerted on the membranous proteins such as ion channels.

The results presented here contribute with basic understanding of mechanisms underlying organisms' tolerance of extreme thermal conditions. Improved understanding of fundamental physiological mechanisms, such as membrane physical properties, underlying acclimation and adaptive processes involved in the thermal biology of ectotherms help to understand responses of ectothermic animals to thermal stress.

\section{References}

[1] H. Bouvrais, M. Holmstrup, P. Westh, J.H. Ipsen, Analysis of the shape fluctuations of reconstituted membranes using GUVs made from lipid extracts of invertebrates, Biol. Open 2 (2013) 373-378.

[2] R.S. Cantor, Breaking the Meyer-Overton rule: predicting effects of varying stiffness and interfacial activity on the intrinsic potency of anesthetics, Biophys. J. 80 (2001) 2284-2297.

[3] M.S. Clark, M.R. Worland, How insects survive the cold: molecular mechanisms - a review, J. Comp. Physiol. B. (2008) 917-933.

[4] S.G. Clerc, T.E. Thompson, Permeability of dimyristoyl phosphatidylcholine/ palmitoyl phosphatidylcholine bilayer membranes with co-existing gel and liquid-crystalline phases, Biophys. J. 68 (1995) 2333-2341.

[5] L. Fernandez-Puente, I. Bivas, M.D. Mitov, P. Méléard, Temperature and chain length effects on bending elasticity of phosphatidylcholine bilayers, Europhys. Lett. 28 (1994) 181-186.

[6] J.R. Hazel, E.E. Williams, The role of alterations in membrane lipid composition in enabling physiological adaptation of organisms to their physical environment, Prog. Lipid Res. 29 (1990) 167-227.

[7] J.M. Hranitz, C.I. Abramson, R.P. Cartera, Ethanol increases HSP70 concentrations in honeybee (Apis mellifera L.) brain tissue, Alcohol 44 (2010) $275-282$.

[8] F.J. Jongeneelen, W.F. Ten Berge, A generic, cross-chemical predictive PBTK model with multiple entry routes running as application in MS Excel; design of the model and comparison of predictions with experimental results, Ann. Occup. Hyg. 55 (2011) 841-864.

[9] A.G. Lee, How lipids affect the activities of integral membrane protein, Biochim. Biophys. Acta 1666 (2004) 62-87.

[10] G. Lindblom, G. Oräd, Lipid lateral diffusion and membrane heterogeneity, Biochim. Biophys. Acta 1788 (2009) 234-244.

[11] C. Montecucco, G.A. Smith, F. Dabbeni-Sala, A. Johansson, Y.M. Galente, R. Bisson, Bilayer thickness and enzymatic activity in the mitochondrial cytochrome c oxidase and ATPase complex, FEBS Lett. 144 (1982) 145-148.

[12] M. Patra, E. Salonen, E. Terama, I. Vattulainen, R. Faller, B.W. Lee, J. Holopainen, M. Karttunen, Under the influence of alcohol: the effect of ethanol and methanol on lipid bilayers, Biophys. J. 90 (2006) 1121-1135.

[13] D. Waagner, M. Holmstrup, M. Bayley, J.G. Sørensen, Induced cold-tolerance mechanisms depend on duration of acclimation in the chill-sensitive Folsomia candida (Collembola), J. Exp. Biol. 216 (2013) 1991-2000. 